Revista de Derecho

Universidad Católica del Norte

Sección: Estudios

Año 18 - No 1, 2011

pp. $21-47$

\title{
REVISIÓN CRÍTICA DEL CRITERIO “INTERÉS DE LA JUSTICIA" COMO RAZÓN PARA NO ABRIR UNA INVESTIGACIÓN O NO INICIAR UN ENJUICIAMIENTO ANTE LA CORTE PENAL INTERNACIONAL ${ }^{*}$
}

\section{Claudia Cárdenas Aravena**}

RESUMEN: El estándar "interés de la justicia” es clave para entender las (necesarias) facultades del Fiscal de la Corte Penal Internacional para la selección de situaciones a investigar entre las que le son remitidas y casos a perseguir cuando ya se ha autorizado la apertura de una investigación. Si bien existen ya varios trabajos sobre el punto, tienen en común que a ellos subyace la comprensión de que dicho estándar se aplicaría en supuestos en los que la investigación de ciertas situaciones y la persecución de ciertos casos esté intrínsecamente en contra del interés de la justicia. Esta interpretación genera varios problemas. Se propone un enfoque, basado en los criterios tradicionales de interpretación de los tratados, que entiende a la cláusula en comento como una válvula de escape en casos de excepción cuyo origen es más bien contingente y externo a la situación o caso en cuestión. Se pone de relieve cómo esta comprensión resulta más coherente con el sistema jurídico internacional en su conjunto.

PaLABRAS CLAVE: Complementariedad - interés de la justicia - Corte Penal Internacional - Fiscal - oportunidad.

\section{REASON FOR NOT STARTING AN INVESTIGATION OR FOR NOT COMMENCING A JUDGEMENT BEFORE THE INTERNATIONAL Criminal Court}

ABSTRACT: The standard "interest of justice" is vital for the understanding of the (necessary) faculties of the prosecutor of the International Criminal Court when selecting the cases for investigations

Este trabajo cuenta con el patrocinio de FONDECYT, proyecto $N^{\circ} 1080060$, denominado "El principio de complementariedad: un cambio de paradigma en la justicia penal internacional". Fecha de recepción: 9 de marzo de 2011.

Fecha de aceptación: 30 de mayo de 2011.

** Magíster y Doctora en Derecho por la Universidad Humboldt de Berlín. Profesora de la Facultad de Derecho de la Universidad de Chile, trabajando actualmente en el programa de Doctorado de la misma Facultad. Correo electrónico: ccardenas@derecho.uchile.cl 
which have been referred and cases to prosecute where it has been authorized to start an investigation. Although there are already several works on the subject matter, they have a common underlying where an understanding that the standard would apply in cases where the investigation of certain situations and the prosecution of certain cases would be inherently against the interest of justice. This interpretation creates several problems. An approach is proposed based on traditional criteria of interpretation of treaties, which interpretes the clause in comment as a form of safety mechanism in exceptional cases whose origin is rather contingent or external to the situation or case. It highlights how this understanding more consistent with the international legal system as a whole.

KEY WORDS: Complementarity - interests of justice - International Criminal Court - prosecutor - opportunity.

\section{INTRODUCCIÓN}

Como se señala en el título, el presente trabajo revisa el criterio "interés de la justicia" como motivo en que el Fiscal de la Corte Penal Internacional puede fundar su decisión de no abrir una investigación o no iniciar un enjuiciamiento penal en los supuestos en los que, conforme al sistema del Estatuto de la Corte Penal Internacional (artículo 53), está jurídicamente facultado para hacerlo.

Esta facultad de selección es importante, ya que no estamos ante un tribunal creado ad hoc para una situación, como todos los tribunales internacionales con competencia penal existentes hasta antes del establecimiento de la Corte Penal Internacional ${ }^{1}$, sino ante un tribunal con competencia de vocación universal. De allí que deba tener una infraestructura normativa que le permita la selección de situaciones y casos según ciertos criterios.

\section{1) RELACIÓN DEL TEMA CON EL PRINCIPIO DE COMPLEMENTARIEDAD}

La relación entre la Corte Penal Internacional y las jurisdicciones nacionales está gobernada por el principio de complementariedad. Conforme a este principio, como es sabido, la Corte no pretende reemplazar

Al respecto, a saber, Mcgoldrick / Rowe / Donnelly (2004). The Permanent International Criminal Court. Legal and policy issues. Oxford and Portland Oregon: Hart Publishing, pp. 14 y ss.; Werle (2005). Tratado de derecho penal internacional. Valencia: Tirant lo Blanch, pp. 49 y ss. 
el sistema preexistente y subsistente de aplicación indirecta del derecho penal internacional por los Estados. Al contrario, viene a complementarlo, teniendo al efecto una competencia material ${ }^{2}$, personal ${ }^{3}$ y temporal ${ }^{4}$ restringida, y estando facultada para actuar solamente en los casos más graves que no hayan sido investigados o perseguidos por otra jurisdicción -cumpliendo con estándares bastante básicos ${ }^{5}$ - y siempre que el crimen de que se trate haya sido cometido en un Estado Parte o por los nacionales de un Estado Parte del Estatuto de la Corte Penal Internacional ${ }^{6}$.

Pues bien, respecto de los casos que caen bajo la competencia de la Corte y que cumplen con los criterios de admisibilidad, vale decir, respecto de los más graves crímenes contra el derecho internacional que no han sido adecuadamente investigados ni perseguidos ${ }^{7}$, el Fiscal puede decidir que no hay fundamento razonable para investigar o perseguir. Si esto ocurre, una Sala de Cuestiones Preliminares de la Corte es informada de la decisión y puede examinar el asunto a solicitud de quien haya remitido la situación -un Estado Parte o el Consejo de Seguridad ${ }^{8}$ y solicitar su reconsideración por parte del Fiscal. Si el Fiscal fundamenta su decisión de no investigar o perseguir solamente en que la investigación o el enjuiciamiento no redundarían en interés de la justicia, la Sala de Cuestiones Preliminares puede revisar la decisión del Fiscal motu proprio. Si lo hace, dicha decisión solo surte efecto si es confirmada por la Sala? .

El hecho de que en caso de que se invoque el interés de la justicia la decisión del Fiscal sea revisable por una Sala de Cuestiones Preliminares de la Corte, implica que la facultad del Fiscal de determinar aquello que ha de estar en interés de la justicia, no puede ser ejercida de manera arbitraria, sino que la decisión debe obedecer a parámetros razonables ${ }^{10}$.

Artículos 5 y siguientes del Estatuto de la Corte Penal Internacional.

Artículos 25 y siguientes del Estatuto de la Corte Penal Internacional.

Artículo 11 del Estatuto de la Corte Penal Internacional.

A saber, Simpson, Ferry (2004). "Politics, sovereignty, rememberance". En Mcgoldrick;

Rowe; Donnelly: The Permanent International Criminal Court. Legal and policy issues.

Oxford and Portland Oregon, Hart Publishing, p. 56.

Artículo 12 del Estatuto de la Corte Penal Internacional.

Artículo 17 del Estatuto de la Corte Penal Internacional.

Artículo 53 párrafo 3 y regla 107 de las Reglas de Procedimiento y Prueba.

Sobre el rol del control jurisdiccional de la decisión del Fiscal, WeI, Wu (2005). Die Rolle des Anklägers eines Internationales Strafgerichtshofs, Fráncfort del Meno: Peter Lang, pp. 191 y ss. Crítico sobre este mecanismo, STIGen, Jo (2008). The relationship between the International Criminal Court and National Jurisdictions: the principle of complementarity. Leiden Boston: Martinus Nijhoff Publishers, pp. 118 y s.

10 Bergsmo, Morten / Kruger, Pieter (2008). “Article 53". En Triffterer, Otto: Commentary on the Rome Statute of the International Criminal Court, $2^{\mathrm{a}}$ ed., Munich: C.H. Beck Hart Nomos, número marginal 22. En general, las facultades del Fiscal de la Corte Penal Internacional están bastante regladas son más estrictas que las del Fiscal en los tribunales ad hoc de los años 90, a saber, Beigbeder, Yves (2005). International justice against impunity. Progress and New Challenges. Boston Leiden: Martinus Nijhoff Publishers, p. 158. 
Ya que, cuando se aplique ese parámetro, la situación o el caso de que se trate no será investigado o perseguido por la Corte, sino que su eventual investigación y persecución queda exclusivamente a cargo de los Estados, la forma en que se entienda que ha de aplicarse el criterio interés de la justicia en el artículo 53 del Estatuto de la Corte Penal Internacional incide en el modo en el cual la jurisdicción de la Corte ha de complementar a la jurisdicción de los Estados para la investigación, persecución y eventual castigo de los crímenes contra el derecho internacional.

\section{2) LAS DISPOSICIONES EN ESTUDIO}

Pasaremos a continuación revista a las dos disposiciones en las que se examinará el estándar "interés de la justicia", para clarificar en qué etapas procesales se aplica.

El trabajo se centra en el significado del interés de la justicia en dos disposiciones, ambas en el artículo 53 del Estatuto de la Corte Penal Internacional: su párrafo 1 letra c) y su párrafo 2 letra c). Ambas dicen relación con las facultades de selección que tiene el Fiscal de la Corte Penal Internacional, primero de situaciones a investigar, luego de casos a perseguir.

Ya que el artículo 53 del Estatuto de la Corte Penal Internacional prevé un mecanismo que puede obligar al Fiscal a investigar y/o a perseguir en ciertos casos, vale decir, fija ámbitos de discrecionalidad y sus límites, se seńalará cuándo, en opinión de la autora, es necesario que el Fiscal justifique su decisión con arreglo al artículo 53 y en qué supuestos esto no resulta necesario, sino que al Fiscal le basta invocar sus facultades discrecionales. Como parámetro general, puede adelantarse que se trata de disposiciones que se aplican excepcionalmente ${ }^{11}$. De ello deriva que no es necesario argumentar que sí concurre el interés de la justicia para cada investigación o enjuiciamiento, pues se presume que es de ese modo ${ }^{12}$. Solo resulta menester fundamentar cuando excepcionalmente este interés no concurre. En cualquier caso, la decisión abrir una investigación o enjuiciamiento puede ser reconsiderada por el Fiscal atendiendo a nuevos hechos o nuevas informaciones conforme al párrafo 4 del artículo 53 del Estatuto de la Corte Penal Internacional.

11 Fiscalía de la Corte Penal Internacional (2007). Policy paper on the interests of justice [fecha de consulta: 9 de febrero de 2011]. Disponible en http://www.icc-cpi.int/NR/ rdonlyres/772C95C9-F54D-4321-BF09-73422BB23528/143640/iccotpinterestsofjustice. pdf

12 En el mismo sentido, Fiscalía de la Corte Penal Internacional (2007) 1 y 3. Parece opinar lo contrario Weвb, Philippa (2005). “The ICC Prosecutor's Discretion not to prosecute in the "interests of justice". Criminal Law Quarterly (50), p. 319. 


\section{(2.1) Artículo 53 párrafo 1 letra C) Del Estatuto de la Cor- TE PENAL InTERnacional}

\section{(2.1.1) Contenido}

Conforme al artículo 53, párrafo 1, letra c) del Estatuto de la Corte Penal Internacional, si el Fiscal estima que la investigación de una situación no redundaría en interés de la justicia, puede decidir que no hay fundamento razonable para abrirla.

Señala el artículo que el Fiscal, luego de examinar la información de que disponga, "iniciará una investigación a menos que determine que no existe fundamento razonable para proceder a ella" de acuerdo al Estatuto. Para decidir, el Fiscal debe tener en cuenta lo siguiente:

a) $\mathrm{Si}$ "[1] a información de que dispone no constituye fundamento razonable para creer que se ha cometido o se está cometiendo un crimen de competencia de la Corte";

b) Si "[l] a causa es o sería admisible";

c) Si hay "razones sustanciales para creer que, aun teniendo en cuenta la gravedad del crimen y los intereses de las victimas, una investigación no redundaría en interés de la justicia".

Vale decir, el Fiscal debe considerar si los hechos caen bajo su competencia material, espacial o temporal ${ }^{13}$. En lo tocante a la admisibilidad, debe dilucidar si el asunto es lo suficientemente grave, y, de estimar que lo es, si está siendo o ha sido investigado o perseguido por otra jurisdicción conforme a ciertos estándares mínimos ${ }^{14}$. En el examen de la letra c), el Fiscal debe volver a considerar la gravedad del asunto (que es grave,

13 Cárdenas, Claudia (2007). "La Corte Penal Internacional y su relación con las jurisdicciones nacionales: El principio de complementariedad". Revista de Magister y Doctorado en Derecho, Universidad de Chile, No 1, pp. 123, 131 y ss. Sobre los crímenes de competencia de la Corte, por todos y con más referencias, Mcgoldrick / Rowe / Donnelly (2004) 123 y ss.; Werle, Gerhard (2005) 309 y ss.; Kittichaisaree, Kriangsag (2001). International Criminal Law. Oxford: Oxford University Press, p. 67 y ss.; Zahar, Alexander / Sluiter, Göran (2008). International criminal law. Oxford: Oxford University Press, p. 109 y ss.

14 Artículo 17 del Estatuto de la Corte Penal Internacional. Por todos, Cárdenas, Claudia (2005). Die Zulässigkeitsprüfung vor dem Internationalen Strafgerichtshof - Zur Auslegung des Art. 17 istgh-Statut unter besonderer Berücksichtigung von Amnestien und Wahrheitskommissionen. Berlín: Berliner Universitätsschriften - Reihe Strafrecht; Gaja, Giorgio (2008). "Issues of admissibility in case of self referrals". En Politi, Mauro / Gioia, Federica: The International Criminal Courts and National Jurisdictions. Adlershot: Ashgate, pp. 49-52; Амвоs, Kai (2010). "El test de complementariedad de la Corte Penal Internacional (art. 17 del Estatuto de Roma”, Indret, № 2. Disponible en www.indret.com [fecha de consulta: 9 de enero de 2011]. 
puesto que es admisible), junto con los intereses de las víctimas ${ }^{15}$, que normalmente serán que se las reconozca como tales, que se vele por no ponerlas nuevamente en riesgo, que se aclaren los hechos y que se obtenga reparación ${ }^{16}$.

Del texto del tratado resulta que el supuesto de la letra c) es claramente excepcional ${ }^{17}$. La expresión "aun teniendo en cuenta" asume que la gravedad y los intereses de las víctimas normalmente favorecerán la apertura de una investigación. Para aplicar esta disposición se requiere que, sin perjuicio de ellos, la investigación no redunde en interés de la justicia. El Fiscal puede invocar esta causal en casos extremos: a un lado de la balanza ha de poner la gravedad de los crímenes y los intereses de las víctimas; al otro, el interés de la justicia. Solo cuando la balanza se incline hacia el interés de la justicia le está permitido no iniciar la investigación. Esta es una facultad privativa de la Oficina del Fiscal, no una obligación; el control por la Sala de Cuestiones Preliminares tiende a prevenir un uso indiscriminado de la facultad del Fiscal, no puede en cambio obligarlo a invocar la cláusula ${ }^{18}$.

El hecho de que se exijan "razones sustanciales" para creer que la investigación no redunda en interés de la justicia implica que no bastan meras apreciaciones sin fundamento, se requiere una base fáctica para la apreciación.

\section{(2.1.2) Ámbito de aplicación}

Una situación puede llegar ante la Corte Penal Internacional por remisión de un Estado Parte, por remisión del Consejo de Seguridad de Naciones Unidas o porque el Fiscal inicia motu proprio una investigación ${ }^{19}$. Si el Fiscal decide no iniciar investigaciones motu proprio, sin que medie remisión, no parece necesario que justifique su decisión ante la Sala de Cuestiones Preliminares, pues actúa simplemente el ejercicio de sus facultades autónomas ${ }^{20}$. Así se desprende por lo demás del artículo 15

15 Por todos, Funk, Markus (2010). Victims rights and advocacy at the International Criminal Court, Oxford: University Press; también WeI (2005) 184 y ss.

16 Estos últimos, conforme al tenor literal de la disposición, son factores independientes de la gravedad, a contrario de lo que seńala CAMEron, Ian (2004). "Jurisdiction and admissibility issues Ander the ICC Statute". En Mcgoldrick / Rowe / Donnelly (2004) 84, que los menciona como criterios guía para determinar lo que ha de entenderse por gravedad.

17 En el mismo sentido Robinson, Darryl (2006). "Serving the interests of justice: Amnesties, truth commissions and the International Criminal Court". En Harrington, Joanna et al.: Bringing power to justice. Québec: Mcgill - Queen's University Press, pp. 210, 217 y ss.

18 Así se corrobora, a saber, en Corte Penal Internacional, decision on the application for leave to appeal the decision on application under rule 103, Situation in Darfur, Sudan, de 4 de febrero de 2009, párrafo 29.

19 Artículo 13 del Estatuto de la Corte Penal Internacional.

20 En el mismo sentido STIGEN (2008) 95 y 111. 
párrafo 6 del Estatuto de la Corte Penal Internacional, donde se señala que si luego del examen preliminar el Fiscal estima "que la información presentada no constituye fundamento suficiente para una investigación, informará de ello a quienes la hubieren presentado". No se prevén otras consecuencias u otro mandato de actuación.

Sin embargo, si la oficina del Fiscal ha recibido remisiones y decide no investigar, debe explicar por qué no lo hace ${ }^{21}$, habida cuenta de que las remisiones por un Estado parte ${ }^{22}$ o por el Consejo de Seguridad son dos de los supuestos que el Estatuto prevé para poner en marcha a la Corte. Esta aproximación resulta acorde con que el Estado remisor y el Consejo de Seguridad de Naciones Unidas, en su caso, puedan pedir a la Sala de Cuestiones Preliminares que revise la decisión del Fiscal ${ }^{23}$.

\section{(2.2) ARTículo 53 PÁRrafo 2 letra C) DEl Estatuto de la CoR- TE PENAL INTERNACIONAL}

\section{(2.2.1) Contenido}

Conforme al artículo 53 párrafo 2 del Estatuto de la Corte Penal Internacional, el Fiscal puede no iniciar un proceso penal si estima, una vez cerrada la investigación, "que no hay fundamento suficiente para el enjuiciamiento", en alguno de los siguientes casos:

a) Si no hay antecedentes suficientes "para pedir una orden de detención o de comparecencia";

b) Si "[l]a causa es inadmisible"; o

c) $\mathrm{Si}$ "[e]l enjuiciamiento no redundaría en interés de la justicia, teniendo en cuenta todas las circunstancias, entre ellas la gravedad del crimen, los intereses de las victimas y la edad o enfermedad del presunto autor y su participación en el presunto crimen".

Para solicitar una orden de detención se requiere, conforme al artículo 58 del Estatuto de la Corte Penal Internacional, que se haya

21 Wouters, Jan / Verhoeven, Sten / Demeyer, Bruno (2009). "The International Criminal Court's Office of the Prosecutor". En Doria, José et al.: The legal regime of the International Criminal Law. Leiden, Boston: Martinus Nijhoff Publishers, pp. 345, 382.

22 La necesidad de dar cuenta del destino de la remisión utilizando los parámetros de este artículo puede constatarse en Corte Penal Internacional, decision requesting information on the status of the preliminary examination of the situation in the Central African Republic, Situation in the Central African Republic, de 30 de noviembre de 2006, p. 4: "Considering that $[\ldots]$ the preliminary examination of a situation pursuant to article 53(1) [...] must be completed within a reasonable time from the reception of a referral by a State Party".

23 Artículo 53 párrafo 3 letra a) del Estatuto de la Corte Penal Internacional. 
iniciado la investigación; que haya motivo razonable para creer que ha cometido un crimen de la competencia de la Corte; y que la detención parezca necesaria para asegurar que la persona comparezca al juicio, para que no obstruya las actuaciones de la Corte o para impedir la comisión de nuevos crímenes. Si la detención no parece necesaria puede solicitarse una orden de comparecencia. Respecto del último de los criterios mencionados en la letra c), puede entenderse como una puerta para que las personas que no estén en condiciones físicas de soportar un proceso no sean juzgadas ${ }^{24}$.

También en esta etapa, a petición del Estado, que ha remitido la situación, o del Consejo de Seguridad de Naciones Unidas, si este la ha remitido, la Sala de Cuestiones Preliminares puede examinar el asunto y solicitar al Fiscal que reconsidere su decisión. Si dicha decisión se basa solamente en el interés de la justicia, la Sala puede revisarla de oficio y únicamente surte efecto si es confirmada ${ }^{25}$.

Distinguiéndolo del relativo a la investigación, el examen previo a iniciar un enjuiciamiento ya no puede ilustrarse tan gráficamente como una balanza, sobre todo porque los factores que se agregan como ejemplos de "todas las circunstancias" -y que tienen que ver con la persona del imputado, que antes no se contemplan, pues estábamos ante una situación y no ante un caso- pueden jugar a favor o en contra de la idea de no perseverar. Así, si estamos ante un imputado con un estado de salud que implica que no sobrevivirá al juicio, malamente podría haber interés de la justicia en juzgarlo, por más atroces que sean los crímenes que se le imputan y por más antecedentes que existan de su participación ${ }^{26}$.

\section{(2.2.2) Ámbito de aplicación}

Estimamos que el artículo 53 párrafo 2 letra c) del Estatuto de la Corte Penal Internacional debe aplicarse cada vez que el Fiscal, habiendo abierto una investigación, decida no perseguir caso alguno respecto de esa situación. En general, el Fiscal tiene la discrecionalidad, no sometida a control, de elegir, dentro de una situación que esté investigando, qué ca-

24 Al respecto STIGEN (2008) 367 y ss., si bien no se refiere exclusivamente a procedimientos ante la Corte Penal Internacional; asimismo Herzog, Felix (2008). "¿No a la persecución penal de dictadores ancianos? Acerca de la función de la persecución penal de la criminalidad estatal". Politica Criminal $\mathrm{N}^{\circ}$ 5, D1, pp. 1-9. Disponible en: www.politicacriminal.cl [fecha de consulta: 9 de febrero de 2011].

25 Hago presente lo que aparece como un error en versión en español del Estatuto de la Corte Penal Internacional si se confronta con las versiones en inglés y en francés: el artículo 53 párrafo 3, cuando se hace referencia a la decisión de proceder, en la versión en español se agrega "a la investigación", lo cual hace que la norma se torne incoherente, pues hace referencia (también) al párrafo 2, que se aplica una vez que la investigación se ha cerrado. 
sos perseguirá27 y cuáles no. Sin embargo, si es que decide no abrir enjuiciamiento alguno una vez que la Corte ya ha puesto sus recursos en una investigación, debe explicar por qué lo hace, teniendo en consideración todas las circunstancias, y su decisión puede ser revisada por la Sala de Cuestiones Preliminares. Así, podría suceder por ejemplo que las personas que se pretendía perseguir hayan muerto o que hayan surgido otras situaciones en las que se hace todavía más urgente el actuar de la Corte. Como es de suponer, en esta etapa el control por la Sala de Cuestiones Preliminares es más fuerte, pues se ha realizado ya inversión recursos humanos y materiales en la investigación de la situación.

\section{3) El parámetro “interés de la justicia” en el Derecho Penal INTERNACIONAL GENERAL}

En los últimos años, sobre todo a propósito de la denominada justicia transicional ${ }^{28}$, abundan las referencias a que en materia penal (también internacional) aparte de la justicia que pudiéramos llamar "ordinaria" cabe prestar atención también otras, que en la literatura reciben el nombre de justicia, entre las que resalta la justicia restaurativa ${ }^{29}$. A fin de ilustrar si esta tendencia ha de tener alguna incidencia a la hora de interpretar la expresión "interés de la justicia" en el Estatuto de la Corte Penal Internacional ${ }^{30}$ resulta menester examinar si acaso ese estándar tiene un significado determinado en materia penal internacional o si, por el contrario, cabe comenzar una interpretación desde cero.

Para lograrlo, lo primero fue identificar todas las disposiciones relevantes en las que se utiliza el criterio de "interés de la justicia", tanto en el

27 Para ello solicitará órdenes de arresto o detención (art. 58 párrafo 2 del Estatuto de la Corte Penal Internacional. Wouters / Verhoeven / Demeyer (2009) 345, 383.

28 Kritz, Neil J. (1996). "Coming to Terms with Atrocities: A Review of Accountability Mechanisms for Mass Violations of Human Rights". Law and Contemporary Problems (59), pp. 127-152; Kritz, Neil J. (ed.) (1995). Transitional Justice. How Emerging Democracies Reckon with Former Regimes, tomo I. Washington: United States Institute of Peace Press. Mcadams A., James (ed.) (1997). Transitional Justice and the Rule of Law in the New Democracies. Notre Dame London: University of Notre Dame Press; Teitel, Ruti G. (2000). Transitional Justice. Oxford: Oxford University Press; Roht-Arriaza, Naomi (1995). "Punishment, redress, and pardon: theoretical and psychological approaches". En: Roht-Arriaza (ed.): Impunity and Human Rights in International Law and Practice. New York oxford: Oxford University Press, pp. 24 y ss.

29 A saber, Villa-Vicencio, Charles (2000). "Restorative justice: dealing with the past differently". En Villa-Vicencio, Charles / Verwoerd, Wilhelm (eds.): Looking back reaching forward. Reflections on the Truth and Reconciliation Commission of South Africa. Cape Town, London: University of Cape Town Press Zed, pp. 68-76.

30 Por una respuesta afirmativa, Yav Katshung, Joseph (2010). The International Criminal Courts and Truth Commissions: two sides of the same coin? Lexington: autoedición, pp. 38 y ss. 
derecho penal internacional como en el derecho internacional de los derechos humanos. A este efecto fueron revisados instrumentos (estatutos, reglas de procedimiento y prueba, otros documentos y jurisprudencia) de la Corte Penal Internacional ${ }^{31}$, del Tribunal Penal Internacional para la ex Yugoslavia ${ }^{32}$, del Tribunal Penal Internacional para Ruanda ${ }^{33}$ y del Tribunal Especial para Sierra Leona ${ }^{34}$, además del Pacto Internacional de Derechos Civiles y Políticos ${ }^{35}$ y el Convenio Europeo sobre Derechos Humanos ${ }^{36}$. En definitiva, se procuró identificar un mínimo común denominador de sentido en estas referencias y alguna interpretación en la jurisprudencia u otros documentos oficiales que pudiera dar luces respecto del modo en que, en el ordenamiento jurídico (penal) internacional, ha de comprenderse la expresión "interés de la justicia".

Al realizar ese examen, se pudo constatar que en la amplísima mayoría de los textos revisados, la expresión se refería a criterios para ejercer facultades o tomar decisiones dentro de un proceso (a saber, regular cuándo se admitirá una prueba, cuándo de asignará un defensor de oficio, cuándo separar procedimientos $)^{37}$. En esta clase de referencias, la expresión "in-

31 Así los siguientes artículos de su Estatuto: 55 párrafo 2 letra c); 61 párrafo 2; 65 párrafo 4; 67 párrafo 1 letra d); sus Reglas de Procedimiento y Prueba 26 párrafo 1; 69; 73 párrafo 6; 76 párrafo 1; 82 párrafo 5; 100 párrafo 1; 136 párrafo 1; 165 párrafo 3; en el Código de Conducta Profesional de los Abogados, artículos 12 párrafo 1 letra b) y 39 párrafo 8 ; el Reglamento de la Corte, artículos 21 párrafo 8; 29 párrafo 1; 54; 60 párrafo 1; 66 párrafo 4; 76 párrafo 1, 80 párrafo 1; Regulations of the Office of the Prosecutor, regulations 29, 31, 33.

32 En su Estatuto artículos 21 párrafo 4 y 28; en sus Reglas de Procedimiento y Prueba, las reglas 3, 4, 15 bis, 15 ter, 44, 45, 45 ter, 53, 62, 70, 71, 73 bis, 73 ter, 79, 81 bis, 82, 85, 89, 93, 108 bis.

33 En su Estatuto, artículo 27.

34 En su Estatuto, artículos 17 párrafo 4 letra d) y 23.

35 Artículo 4.

36 Artículo 6.

37 Solo a título ejemplar, Corte Penal Internacional: Decision ordering the parties to submit their observations on the applications for Victims' participation in the proceedings, Prosecutor v. Omar Hassan Ahmad Al Bashir, de 1 de septiembre de 2009, p. 4; CorTe Penal Internacional: Decision on legal representation, appointment of counsel of defence, Prosecutor v. Kony et al., de 17 de septiembre de 2008, p. 7; Corte Penal InterNACIONAL: Decision on the joinder of the Cases against Germain Katanga and Mathieu Ngudjolo Chui, Prosecutor v. Germain Katanga, de 10 de marzo de 2008; Tribunal Penal Internacional para la ex Yugoslavia: Decision on request to admit additional evidence, Prosecutor v. Goran Jelisic, de 15 de noviembre de 2000; Tribunal Penal Internacional para la ex Yugoslavia: Decision on assignment of defence counsel, Prosecutor v. Vesselin Sljivancanin, de 20 de agosto de 2001; Tribunal Penal Internacional para la ex YuGOSLAVIA: Decision on appellants motion for the extension of the time-limit and admission of additional evidence, Prosecutor v. Dusko Tadic, de 15 de octubre de 1998; Tribunal Penal Internacional para la ex Yugoslavia: Decision on presecution's motion for order appointing counsel to assist Vojislav Seselj with his defence, Prosecutor v. Vojislav Seselj, de 9 de mayo de 2003; Tribunal Penal Internacional para la ex Yugoslavia: Decision on the admission of additional evidence following hearing of 30 march 2001, Prosecutor $v$. Kupreskic et al., de 11 de abril de 2001; Tribunal Penal Internacional para la ex Yugoslavia: Decision on assignment of defence counsel, Prosecutor v. Isak Musliu, de 21 de 
terés de la justicia" bien podría ser reemplazada por intereses del proceso (debido, justo y racional). Se utiliza para expresar la mejor forma de satisfacer de manera expedita y confiable los fines del proceso en sentido estricto (investigar el crimen, la participación y determinar el castigo) ${ }^{38}$, pues esa la forma en que se está llevando a cabo la justicia. Siguiendo a Stigen, la versión rusa del Estatuto iría en el mismo sentido - "interesty pravosudia" se traduciría usualmente como interés del tribunal, o interés de la administración de justicia-, lo que es particularmente relevante si se tiene presente que la versión rusa está entre las vinculantes del Estatuto de la Corte Penal Internacional conforme a su artículo $128^{39}$.

El principal hallazgo de esta parte de la investigación es que la expresión "interés de la justicia" no resulta extraña al derecho (penal) internacional, por lo que la interpretación a la que se llegue en esta trabajo ha de ser compatible con el uso que se da a la expresión "interés de la justicia" en contextos distintos del artículo 53 del Estatuto de la Corte Penal Internacional, que coincide ampliamente con los intereses del proceso.

\section{4) ENFOQUE HASTA AHORA PREDOMINANTE EN LA INTERPRETACIÓN DEL INTERÉS DE LA JUSTICIA EN EL ARTÍCULO 53 DEL ESTATUTO DE la Corte Penal InTERnacional}

Examinando lo publicado hasta la fecha sobre el tópico "interés de la justicia” en el artículo 53 del Estatuto de la Corte Penal Internacional, puede constatarse que las aproximaciones suelen examinar en qué casos

octubre de 2003; Tribunal Penal Internacional para la ex Yugoslavia: Decision on independent counsel for Vidoje Blagojevic's motion to instruct the registrar to appoint new lead and co-counsel, Prosecutor v. Blaojevic et al., de 3 de julio de 2003; Tribunal Penal Internacional para la ex Yugoslavia: Decision on appeal by Bruno Stojic against Trial Chamber's decision on request for appointment of counsel, Prosecutor $v$. Prlic et al., de 24 de noviembre de 2004; Tribunal Penal Internacional para la ex Yugoslavia: Decision on assigned counsel's motion for withdrawal, Prosecutor v. Slobodan Milosevic, de 7 de diciembre de 2004; Tribunal Penal Internacional para la ex Yugoslavia: Decision on the prosecution motion for receiving testimony by video-conference link, Prosecutor $v$. Hadzihasanovic et al., de 11 de marzo de 2004; Tribunal Penal Internacional para la ex Yugoslavia: Order separating proceedings and scheduling order, Prosecutor $v$. Simic et al., de 24 de enero de 2001. Solo un grupo muy menor de referencias -el artículo 53 del Estatuto de la Corte Penal Internacional, y disposiciones sobre indulto en los estatutos de los tribunales ad hoc-se referían a situaciones que ocurren antes y después, respectivamente, del proceso penal.

38 Glaser, Juluis (1883). Handbuch des Strafprozesses, primer tomo. Leipzig: Duncker \& Humblot, p. 1; Roxin, Claus (1998). Strafverfahrensrecht. Munich: Beck, número marginal 3 y ss. En el mismo sentido Federation Internationale des Ligues des Droits De L'номме (2005). Reflexions sur la notion "interets de la justice" au terme de l'article $53 d u$ Statut de Rome. Disponible en http://www.fidh.org/IMG/pdf/cpi20062005f.pdf [fecha de consulta: 9 de enero de 2010].

39 Stigen (2008) 355. 
podría afirmarse que la investigación o persecución de ciertos asuntos podría, por sí misma, contravenir el interés de la justicia. Los diversos autores señalan cuándo ellos creen que se daría este supuesto. Si bien existen enfoques más o menos estrictos, según los casos, se reiteran en doctrina las referencias a las comisiones de verdad y reconciliación, a las amnistías (justicia retributiva versus justicia restaurativa) y a los procesos de $\mathrm{paz}^{40}$, ya sea para expresar su opinión de incluirlas en los casos en los que una investigación o persecución penal contraría a los intereses de la justicia o de no hacerlo ${ }^{41}$. En cualquier caso, teniendo -reitero- aproximaciones más o menos estrictas, subyace la idea de que en los supuestos en los que no se cumple con los estándares para que la persecución y castigo quede intrínsecamente fuera del interés de la justicia, el Fiscal deberá proceder a la investigación o a la persecución. Por lo tanto, como consecuencia lógica, en esos supuestos la Sala de Cuestiones Preliminares debería objetar su decisión de no investigar o perseguir, según corresponda.

40 Se entiende la referencia si se tiene en mente que la Corte Penal Internacional actuará usualmente en situaciones en las que será necesario negociar la paz, Bensouda, Fatou (2010). "Challenges related to investigation and prosecution at the International Criminal Court". En Belleli, Roberto (ed.) (2010): International Criminal Justice. Surrey: Ashgate, pp. 131, 141 y ss.; Arsanjani, Mahnoush (1999). "Reflections on the jurisdiction and trigger mechanism of the International Criminal Court". En Von Hebel, Hermann / Lammers, Johan / Schukking, Jolien: Reflections on the International Criminal Court. La Haya: T.M.C. Asser Press, pp. 57, 75 y ss.; Razesberger, Florian (2006). The International Criminal Court. Fráncfort del Meno: Peter Lang, p. 176; Robinson (2006) 210, 220 y ss.; STigen (2008) 358, 431 y ss.

41 Goldstone, Richard J. / Fritz, Nicole (2000). “'In the interests of justice' and independent referral: The ICC prosecutor's unprecedent powers". Leiden Journal of International Law, No 13, pp. 355 y ss. Ellos entienden que amnistías que están de acuerdo con ciertos parámetros pueden ser consistentes con la justicia, lo cual parece en cualquier caso quedar excluido para los crímenes de competencia de la Corte. De manera similar en lo restrictivo razona Stahn, Carsten (2005). "Complementarity, Amnesties and alternative forms of justice: some interpretative guidelines for the International Criminal Court". Journal of International Criminal Justice, No 3, pp. 695, 719: "The Statute leaves some room to recognize amnesties and pardons, where they are conditional and accompanied by alternative forms of justice which may lead to prosecution". Webв (2005) 338 y ss.; Scharf, Michael (1999). "The amnesty exception o he jurisdiction of the International Criminal Court". Cornell International Law Journal, No 32, pp. 509, 524. Valińa requiere que haya "a certain process of accountability", no completa impunidad. VALIÑA, Marta (2010). "Interpreting complementarity and Interests of justice in the presence of restorative-based alternative forms of justice". En Stahn, Carsten / Van den Herik, Larissa: Future perspectives of international criminal justice. La Haya: T.M.C. Asser Press, pp. 167, 269, refuerza la idea de casos muy excepcionales, equiparables a un estado de necesidad en p. 277. Seńala criterios específicos a considerar en p. 287; Brubacher, Matthew (2004). "Prosecutorial discretion within the International Criminal Court". Journal of International Criminal Justice, № 2, pp. 71, 81. 


\section{5) Problemas de la interpretación aCtUal}

Es posible identificar algunos problemas que se suscitan con la aproximación predominante hasta ahora, consistente en buscar, en el artículo 53, párrafo 1 letra c) del Estatuto de la Corte Penal Internacional, una interpretación de la expresión "interés de la justicia" basada en los supuestos en los que la investigación y persecución de ciertos crímenes internacionales podrían ir intrínsecamente contra ese interés:

1. No es claro, menos aún en la lógica del establecimiento de la Corte Penal Internacional ${ }^{42}$, que haya crímenes internacionales cuya investigación y persecución no esté intrínsecamente en interés de la justicia $^{43}$, con la sola salvedad de la persecución de personas físicamente incapaces de soportar un juicio.

2. La aproximación que observamos en la literatura no discute el problema al que puede verse enfrentado el Fiscal en caso de una sobrecarga de trabajo que pueda impedirle llevar a cabo su labor adecuadamente. De hecho, como ya quedó mencionado, subyace la idea de que si no se cumplen los estándares para que la persecución y castigo quede intrínsecamente fuera de los intereses de la justicia, el Fiscal deberá proceder a la investigación o a la persecución, según los casos. Se hace de este modo caso omiso a que lo que se ha de llevar adelante no es cualquier procedimiento, sino uno que resguarde ciertas garantías mínimas. En la literatura disponible no es frecuente encontrar referencias al punto de los recursos materiales, y cuando existen no se lo trata como aspecto que puede llegar a ser determinante, sino que se dice expresamente que no debiese ser un criterio decisivo ${ }^{44}$.

42 Preámbulo de su Estatuto, párrafos 4 y 5 y en doctrina, Olásolo, Héctor (2003). Corte Penal Internacional ¿¿Dónde investigar? Valencia: Tirant lo Blanch, p. 259 y ss.

43 En la misma línea Sunga, Lyal S. (2009). "Ten principles for reconciling truth commissions and criminal prosecutios". En Doria, José / Gasser, Hans-Peter / Bassiouni, M. Cherif: The legal regime of the International Criminal Court. Leiden. Boston: Martinus Nijhoff Publishers. pp. 1071, 1101: "To avoid sending mixed messages to would-be violators, the ICC Prosecutor should investigate all situations where there is a reasonable basis to do so, regardless as to whether there is an amnesty in place, or negotiations to arrange one". "Interestingly in this regard, after many years of waging a bloody insurgency and committing severe violations in Uganda, Joseph Kony suddenly started talking peace only once the ICC prosecutor proceeded to investigate allegations of Kony's criminal responsibility".

$44 \quad$ Weвв (2005) 342: "In sum, the amount of financial and temporal resources that a trial would use should be a criterion in assessing the 'interests of justice', but it should not be a decisive criterion. Efficiency is a worthy goal, but it is not the purpose of the ICC". También Webв (2005) 340 y ss.; Behrens, H. J. (1999). “Investigación, juicio y apelación”. En Ambos, Kai / Guerrero, Julián (comp.): El Estatuto de la Corte Penal Internacional. Bogotá: Universidad Externado de Colombia, p. 319, criticado por Gómez Colomer, José Luis (2003). "La investigación del crimen en el proceso penal ante la Corte Penal Internacional”. En Gómez Colomer, José Luis / González Cusac, José Luis / Cardona Llorens, Jorge (coords.): La Corte Penal Internacional. Valencia: Tirant lo Blanch, pp. 300 y ss. 
3. Esta interpretación tampoco entrega herramientas para enfrentar problemas como el que el caso Lubanga ha mostrado que pueden presentarse ante la Corte Penal Internacional, vale decir, que una persona esté siendo perseguida por crímenes graves en su Estado de origen, sin perjuicio de que el caso que pretende perseguir la Corte es admisible porque no está siendo perseguido ${ }^{45}$.

4. Con esta aproximación no se entiende por qué el examen acerca del interés de la justicia ha de llevarse adelante en dos niveles (primero previo a abrir la investigación y luego previo al enjuiciamiento), reiterando parcialmente los parámetros. No resulta comprensible cómo, si una investigación por la Corte Penal Internacional estuvo intrínsecamente en interés de la justicia, iniciar al menos un enjuiciamiento en esa situación podría no estarlo, bajo los criterios de gravedad e intereses de las víctimas, que han de utilizarse obligatoriamente en ambas etapas.

\section{6) INTERPRETACIÓN PROPUESTA}

Después de examinar los alcances de las disposiciones en juego y la interpretación hasta ahora mayoritaria en doctrina, la propuesta fundamental de este trabajo radica en entender que la cláusula "interés de la justicia” es una herramienta de selección de situaciones y casos para la Fiscalía, que se basa en criterios contingentes y eventualmente externos a la situación o caso, vale decir, al aplicarla no se predica que investigar esa situación o perseguir ese caso contraríen el interés de la justicia en términos generales, sino solamente en las circunstancias dadas.

Seguir lo planteado en esta propuesta permite una nueva aproximación a todos los problemas detectados desde la interpretación anterior ${ }^{46}$. Así, respetando la enumeración del acápite anterior, sigue una exposición de los puntos allí expuestos desde la perspectiva de la aproximación propuesta.

45 No lo será si se persigue a la misma persona por la misma conducta (el denominado "same conduct test" que ha sido recogido en diversas resoluciones de la CPI desde Corte Penal Internacional: Decision on the Prosecutor's application for warrants of arrest, article 58, Situation in the Democratic Republic of Congo, de 10 de febrero de 2006, párrafo 38.

46 Incluso suprime dificultades de lectura como las que pone de manifiesto Gallavin, Chris (2003). "Article 53 of the Rome Statute of the International Criminal Court: in the interests of justice?”. King's College Law Journal, No 179, pp. 179, 185 y ss. 


\section{(6.1) ARMONÍA CON EL DERECHO INTERNACIONAL EN SU CONJUNTO}

La interpretación propuesta da al artículo 53 del Estatuto de la Corte Penal Internacional un sentido que está en conformidad con las demás disposiciones del Estatuto y con el derecho internacional general. Teniendo en cuenta las reglas comunes de interpretación de los tratados, al interpretar sus disposiciones debe tenerse en cuenta su texto, su contexto, su objeto y fin ${ }^{47}$, y procurar que su texto tenga un efecto útil ${ }^{48}$. Aplicando estos criterios a la interpretación del artículo 53 del Estatuto de la Corte Penal Internacional, ellos no dan luces respecto de que la persecución de los crímenes más graves contra el derecho internacional pueda no estar intrínsecamente en interés de la justicia, sino por el contrario, el derecho internacional general y el Estatuto de la Corte Penal Internacional prescriben que deben ser perseguidos. Téngase en cuenta, a saber, la máxima aut dedere aut judicare, y lo manifestado el preámbulo del Estatuto de la Corte Penal Internacional ("los crimenes más graves de trascendencia para la comunidad internacional en su conjunto no deben quedar sin castigo". "Decididos a poner fin a la impunidad de los autores de esos crimenes" ${ }^{49}$. Si esas ideas inspiraron el establecimiento de la Corte, fue justamente pensando en que para concretarlas la Corte debe funcionar, llevando adelante investigaciones y procesos. Ello está en consonancia con el sentido que otros artículos del Estatuto, las Reglas de Procedimiento y Prueba, las Regulaciones de la Corte Penal Internacional y otras disposiciones ${ }^{50}$, dan a la expresión "interés de la justicia”, vale decir, los intereses de un justo y racional procedimiento o el interés de cumplir adecuadamente con los fines del proceso, según los casos.

Suele sostenerse que el quehacer de la Corte puede reemplazarse válidamente por otros medios, como comisiones de verdad y reconciliación $^{51}$ e incluso amnistías en determinados supuestos. Por los fines que persiguen, las comisiones de verdad y reconciliación pueden ser medios complementarios al proceso penal para tratar, como sociedad, una determinada situación. Con todo, su existencia no ha se ser un obstáculo para

47 Fiscalía de la Corte Penal Internacional (2007) 1 y 4, en concordancia con el artículo 31 de la Convención de Viena sobre el Derecho de los Tratados.

48 En el mismo sentido Fiscalía de la Corte Penal Internacional (2007).

49 En el mismo sentido y aún más tajante, Olásolo (2003) 259 y ss. El artículo 53 párrafo 1 se refiere a que exista un fundamento razonable para proceder "conforme al Estatuto" (con protección debida de testigos y demás estándares). GaLlavin (2003) 179, 185 y ss.

50 A saber, el artículo 14 del Pacto Internacional de Derechos Civiles y Políticos como razón para que una audiencia no sea pública. Lo mismo se dispone en el artículo 8 párrafo 5 del Pacto de San José.

51 Sobre estas comisiones, entre la abundante bibliografía, confróntese por todos Hayner, Priscilla (2002). Unspeakable truths. Nueva York, Londres: Routledge; Rotвerg, Robert / Thomson, Dennis (eds.) (2000). Truth versus justice. Princeton: Princeton University Press. 
la justicia penal, ni afecta por sí sola el interés de la justicia en la investigación o persecución de crímenes contra el derecho internacional. Actuar asumiendo lo contrario acarrea el riesgo que la eventual investigación o persecución por la Fiscalía de la Corte pase a ser una de las materias en la mesa de negociación, lo que dicha Fiscalía ha rechazado ${ }^{52}$. Teniendo a la vista que los bienes jurídicos protegidos por el derecho penal internacional son la paz, la seguridad y el bienestar de la humanidad, la eventual existencia de comisiones de verdad y reconciliación es importante, pero no reemplaza, por sus fines, a la persecución penal ${ }^{53}$. Si basándonos en el mismo Estatuto, comprendemos, siguiendo al preámbulo, que el derecho penal internacional persigue -a lo menos también- fines absolutos, no queda más que constatar que habrá que perseguir para satisfacerlos. Contingencias pueden hacer que en un momento deban seleccionarse situaciones a investigar y casos a perseguir entre aquellos que son de la competencia de la Corte y cumplen con los estándares de admisibilidad. Esa selección no implica sostener que la investigación o la persecución de las situaciones o casos no seleccionados no interesa a la justicia, pues en el sistema del Estatuto de la Corte Penal Internacional dicho interés está del lado de la investigación y persecución de todos los crímenes contra el derecho internacional.

\section{(6.2) PeRMite haCERSE CARgo DEL PROBleMA DE LOS RECURSOS LI- MITADOS}

Esta aproximación toma debidamente en consideración un hecho que a estas alturas no debiera pasar desapercibido: la Corte en general, y la Fiscalía en particular, tienen recursos limitados, que en ningún caso alcanzan para investigar y perseguir todas las situaciones y los casos admisibles. ¿Qué hacer entonces? Si se considera que en caso de sobrecarga de trabajo el Fiscal no puede utilizar la válvula de escape doblemente excepcional del artículo 53 párrafos 1 letra c) y 2 letra c), se lo estaría obligando a actuar en más situaciones o casos de los que puede abarcar con propiedad. Ese escenario de una Corte trabajando por sobre su capacidad, redundaría en que con toda probabilidad no se podría cumplir con los estándares que exigen las complejas situaciones y los complejos casos que

52 Fiscalía de la Corte Penal Internacional (2005). Second public hearing of the Office of the Prosecutor [fecha de consulta: 9 de febrero de 2011]. Disponible en: http:// www.icc-cpi.int/Menus/ICC/Structure + of + the + Court/Office + of + the+Prosecutor/ Network+with+Partners/Public+Hearings/Second+Public+Hearing/Session+1/Opening+Sta tement+by+the+Chief+Prosecutor.htm, p. 21.

53 CÁrdenas (2005) 172 y ss. Sosteniendo que los procesos judiciales son más idóneos para lograr la reconciliación, Murphy, Colleen (2010). "Political reconciliation and international criminal trials". En May, Larry / Hoskins, Zachary: International criminal law and philosophy. Cambridge: Cambridge University Press, pp. 236 y ss. 
caen bajo la competencia de la Corte. Ello dañaría tanto a las víctimas como a la imagen de legitimidad de la justicia internacional -que no solamente debe mostrar legitimidad de origen, sino también de ejercicio ${ }^{54}$. Si la Corte llegara a colapsar, esto iría en contra del objetivo declarado de los Estados Partes de "garantizar que la justicia internacional sea respetada $y$ puesta en práctica en forma duradera". Este escenario iría abiertamente en contra del interés de la justicia, tanto en general como para cada una de las situaciones y cada uno de los casos involucrados. La nueva forma de aproximación a la fórmula en comento, que proponemos, fuera de abarcar los casos en los que una investigación o enjuiciamiento puedan estar intrínsecamente fuera del interés de la justicia, abarca los supuestos en los que en abstracto merecerían investigación y proceso, pero en los que en la práctica no es posible hacerlo por la Corte en ese momento sin detrimento del trabajo de la Corte en general. El Fiscal de la Corte Penal Internacional no puede estar obligado a lo imposible (impossibilium nulla obligatio est $)^{55}$.

Así, si bien la investigación de una situación o la persecución de un caso pudiera estar intrínsecamente en consonancia con los intereses de la justicia, su investigación o persecución en el momento de que se trate, por parte de la Corte, podría ir en contra del objeto y fin del tratado, de llevar adelante procesos con garantías y altos estándares para juzgar y eventualmente castigar los crímenes de trascendencia internacional más graves.

54 Sobre la legitimidad en el derecho internacional, Meyer, Lukas H (ed.) (2009): Legitimacy, justice and public international law. Cambridge: Cambridge University Press; Wolfrum, Rüdiger / Röвen, Volker (eds.) (2008): Legitimacy in international law. Berlín, Heidelberg, Nueva York: Springer; CLARK, Ian (2007). International legitimacy and world society. Oxford: Oxford University Press; Clark, Ian (2005). Legitimacy in international society. Oxford: Oxford University Press. En particular en el ámbito de la Corte Penal Internacional Struett, Michael J. (2009). "The politics of discursive legitimacy: understanding the dynamics and implications of prosecutorial discretion at the International Criminal Court". En Roach, Steven C.: Governance, order and the International Criminal Court. Oxford: Oxford University Press), p. 151 y ss.; Struett, Michael J. (2008). The Politics of constructing the International Criminal Court. New York: Palgrave Macmillan.

55 Fiscalía de la Corte Penal Internacional (2005) 19: "[I]n establishing criteria for the selection of incidents, cases and charges, the OTP, guided by the terms of the Rome Statute, must endeavour to bridge the gap between what ideal justice demands and what resources and practicability dictate"; LeE, Roy (1999). The International Criminal Court. The making of the Rome Statute. The Hague, London, Boston: Kluwer Law International; Guariglia, Fabricio (1999). "Investigation and prosecution". En Triffterer; Otto (ed.): Commentary on the Rome Statute of the International Criminal Court. Baden-Baden: Nomos, pp. 227, 230. 


\section{(6.3) ENTREGA HERRAMIENTAS PARA PRIORIZAR LOS PROCEDIMIEN- TOS ESTATALES POR CRÍMENES IGUALMENTE GRAVES CUANDO LA ADMISIBILIDAD NO ESTÁ EN JUEGO}

Con la interpretación propuesta no habría inconvenientes en aplicar la cláusula si un Estado está juzgando casos al menos de igual gravedad que los que pretende juzgar la Corte Penal Internacional, con estándares mínimos.

Si un Estado está juzgando, en un proceso que cumple con las garantías mínimas, a la misma persona por otros crímenes a lo menos de igual gravedad que los investigados por la Corte Penal Internacional, no parece adecuado al interés de la justicia ponerlo en posición de solicitarle que entregue al imputado, por lo menos hasta que termine el juicio y cumpla su condena. Esta aseveración se basa en el consenso existente en torno a las ventajas de la persecución estatal por sobre la internacional, sobre todo si juzga el Estado territorial: se avizoran menores dificultades idiomáticas, mayor familiaridad del imputado con el sistema jurídico, mayor cercanía con los medios de prueba y se posibilita de mejor manera cerrar el círculo del conflicto dentro de la propia sociedad antes conflictuada, por los mecanismos que ella se ha dado ${ }^{56}$.

Aplicando el artículo 53 del Estatuto de la Corte Penal Internacional podría permitirse entonces que el procedimiento ante la Corte Penal Internacional se aplace hasta el final del juicio y eventual cumplimiento de la condena, aunque el caso de que se trate sea admisible por tratarse de una conducta que no está siendo objeto de investigación ni enjuiciamiento en el Estado. Sería esta una manifestación particularmente elocuente del principio de complementariedad.

\section{(6.4) ES COMPATIBLE CON LA DOBLE APLICACIÓN DE ESTÁNDARES}

Esta interpretación -que se basa en un examen contingente- es compatible con que se examine en dos ocasiones, primero para una situación y luego para casos dentro de esa situación, los factores de gravedad y de intereses de las víctimas.

Si se tratara de un examen destinado a determinar si la investigación de una situación o la persecución de un caso están intrínsecamente en interés de la justicia, sería a lo menos difícil de explicar que si una investigación estuvo en interés de la justicia considerando los criterios de gravedad e intereses de las víctimas, un examen que incluye a los mismos factores, como lo manda el artículo 53 párrafo 1 letra c), lleve a concluir 56 Tomuschat, Christian (1998). "Das Statut von Rom für den Internationalen Strafgerichtshof”.
Die Friedenswarte, pp. 335, 342. 
que contraría al interés de la justicia abrir al menos una persecución en dicha situación. En cambio, desde la aproximación propuesta, tomando a la gravedad como criterio relevante al decidir si se inicia una persecución, parece ser que en el universo de casos graves, habida cuenta que la Corte no tiene la capacidad de recursos para perseguirlos a todos, debe elegir, y esa elección debe hacerla teniendo en cuenta, entre otros parámetros, la gravedad, procurando elegir a los más graves. Algo análogo ocurre con el grado de responsabilidad. No puede decirse que esté en interés de la justicia que a quienes no están entre los más responsables no se los persiga, sino que está en interés de la justicia que los casos que la Corte siga los lleve a buen término seriamente. Para eso es necesario que seleccione, ya que no tiene capacidad para atenderlos a todos. La selección está en interés de la justicia y el Estatuto da criterios para llevarla a cabo ${ }^{57}$.

\section{Palabras finales}

En resumen, la principal diferencia de la lectura que proponemos con la doctrina dominante es que postulamos que la investigación de las situaciones o la persecución de los casos no seleccionados conforme al artículo 53 del Estatuto de la Corte Penal Internacional no necesariamente está contra el interés de la justicia como consecuencia de características intrínsecas que les son propias, sino más bien que la presencia o ausencia del interés de la justicia en un supuesto determinado puede tener origen en circunstancias distintas de la situación o el caso concreto y sus circunstancias inmediatas. Se sostiene que la decisión de no investigar o no perseguir no implica necesariamente que el investigar esa situación o perseguir ese caso no redunde, en términos absolutos, en interés de la justicia.

De manera coherente con la naturaleza contingente de la estimación, la decisión de no proceder no es definitiva en el tiempo ${ }^{58}$, lo cual coincide asimismo con la naturaleza imprescriptible de los crímenes relevantes ${ }^{59}$. En doctrina se han formulado reparos a que la duración de los procedimientos ante la Corte se extiendan en exceso ${ }^{60}$, sin embargo en los estudios en comento todavía no se dirige el proceso contra personas en concreto, por lo que estimamos que no sería aplicable ese reparo a la posibilidad de posterior revisión de la decisión de no investigar o no perseguir.

57 Sobre la normativa del artículo 53 Estatuto de la Corte Penal Internacional como una positivización del principio de oportunidad en el procedimiento ante la Estatuto de la Corte Penal Internacional, WeI (2005) 177 y ss.

Artículo 53 párrafo 4 Estatuto de la Corte Penal Internacional.

Artículo 29 Estatuto de la Corte Penal Internacional.

Gioia, Federica (2008). "The complementarity role of the International Criminal Court; are they any time limits?" En Politi, Mauro / Gioia, Federica: The International Criminal Courts and National Jurisdictions. Adlershot: Ashgate, pp. 71-80. 
En el Estatuto se exige que el Fiscal actúe razonablemente en la selección de situaciones y casos, utilizando parámetros que lleguen a convencer a la Sala de Cuestiones Preliminares. No parece necesario establecer a priori los parámetros a utilizar, pues la disposición en su conjunto está pensada para ser utilizada en situaciones de excepción que habrá que considerar en cada caso ${ }^{61}$, sin desmerecer la necesidad de justicia en las demás situaciones y casos cuya investigación o persecución se descarta. Es la racionalidad de la selección la que va a medir la Sala. Dentro de parámetros razonables, la discrecionalidad en la materia corresponde a la Fiscalía $^{62}$.

\section{BIBLIOGRAFÍA}

- Ambos, Kai (2010): "El test de complementariedad de la Corte Penal Internacional (art. 17 del Estatuto de Roma” en Indret 2/2010. Disponible en www.indret.com [fecha de consulta: 9 de enero de 2011].

- Arsanjani, Mahnoush (1999): "Reflections on the jurisdiction and trigger mechanism of the Internacional Criminal Court". En Von Hebel, Hermann; Lammers, Johan; Schukking, Jolien: Reflections on the International Criminal Court. La Haya: T.M.C. Asser Press.

- Beigbeder, Yves (2005): International justice against impunity. Progress and New Challenges. Boston Leiden: Martinus Nijhoff Publishers.

- Behrens, H.J. (1999): "Investigación, juicio y apelación”. En Ambos, Kai / Guerrero, Julián (compiladores): El Estatuto de la Corte Penal Internacional. Bogotá: Universidad Externado de Colombia.

- Bensouda, Fatou (2010): "Challenges related to investigation and prosecution at the International Criminal Court". En Belleli, Roberto (editor) (2010): International Criminal Justice. Surrey: Ashgate.

- Bergsmo, Morten; Kruger, Pieter (2008): “Article 53". En Triffterer; Otto: Commentary on the Rome Statute of the International Criminal Court, 2a ed. Munich: C. H. Beck Hart Nomos.

\footnotetext{
61 Schabas; William (2010). The International Criminal Court: a commentary on the Rome Statute. Oxford: Oxford University Press, p. 663. A título ejemplar, consúltese el esfuerzo de enumeración de STIGEN (2008) 372 y ss.

62 En este punto de acuerdo con Schabas (2010) 748.
} 
- Brubacher, Matthew (2004): "Prosecutorial discretion within the International Criminal Court". Journal of International Criminal Justice (2).

- Cárdenas, Claudia (2007): "La Corte Penal Internacional y su relación con las jurisdicciones nacionales: El principio de complementariedad“. Revista de Magister y Doctorado en Derecho, Universidad de Chile, No 1/2007.

- CÁrdenas, Claudia (2005). Die Zulässigkeitsprüfung vor dem Internationalen Strafgerichtshof - Zur Auslegung des Art. 17 IStGHStatut unter besonderer Berücksichtigung von Amnestien und Wahrheitskommissionen. Berlín: Berliner Universitätsschriften Reihe Strafrecht 24.

- Clark, Ian (2007). International legitymacy and world society. Oxford: Oxford University Press.

- Clark, Ian (2005). Legitymacy in international society. Oxford: Oxford University Press.

- Federation Internationale des Ligues des Droits de L'homme (2005). Reflexions sur la notion "interets de la justice" au terme de l'article 53 du Statut de Rome. Disponible en http://www.fidh.org/ IMG/pdf/cpi20062005f.pdf [Fecha de consulta: 9 de enero de 2010].

- Funk, Markus (2010). Victims rights and advocacy at the International Criminal Court Oxford: University Press.

- Gaja, Giorgio (2008). "Issues of admissibility in case of self referrals". En Politi, Mauro / Gioia, Federica: The International Criminal Courts and National Jurisdictions. Adlershot: Ashgate.

- Gallavin, Chris (2003). "Article 53 of the Rome Statute of the International Criminal Court: in the interests of justice?". King's College Law Journal (179).

- Giora. Federica (2008). "The complementarity role of the International Criminal Court; are they any time limits?" En Politi, Mauro / Gioia, Federica: The International Criminal Courts and National Jurisdictions. Adlershot: Ashgate.

- Glaser, Juluis (1883). Handbuch des Strafprozesses, primer tomo. Leipzig: Duncker \& Humblot.

- Goldstone, Richard J. / Fritz, Nicole (2000). "In the interests of justice' and independent referral: The ICC prosecutor 's unprecedent powers". Leiden Journal of International Law (13).

- Gómez Colomer, José Luis (2003). "La investigación del crimen en el proceso penal ante la Corte Penal Internacional”. En Gómez Colomer, José Luis / González Cusac, José Luis, Cardona Llorens, Jorge (coordinadores): La Corte Penal Internacional. Valencia: Tirant lo Blanch. 
- Guariglia, Fabricio (1999). "Investigation and prosecution". En Triffterer; Otto (editor): Commentary on the Rome Statute of the International Criminal Court. Baden-Baden: Nomos.

- Hayner, Priscilla (2002). Unspeakable truths. Nueva York, Londres: Routledge.

- Herzog, Felix (2008). “¿No a la persecución penal de dictadores ancianos? Acerca de la función de la persecución penal de la criminalidad estatal". Politica Criminal $\mathrm{N}^{\circ}$ 5, D1, pp. 1-9. Disponible en: www.politicacriminal.cl [fecha de consulta: 9 de febrero de 2011].

- Kittichaisaree, Kriangsag (2001). International Criminal Law. Oxford: Oxford University Press.

- Kritz, Neil J. (1996). "Coming to Terms with Atrocities: A Review of Accountability Mechanisms for Mass Violations of Human Rights". Law and Contemporary Problems (59).

- Kritz, Neil J. (ed.) (1995). Transitional Justice. How Emerging Democracies Reckon with Former Regimes, tomo I. Washington: United States Institute of Peace Press.

- Lee, Roy (1999). The International Criminal Court. The making of the Rome Statute. The Hague, London, Boston: Kluwer Law International.

- McAdams, A. James (ed.) (1997). Transitional Justice and the Rule of Law in the New Democracies. Notre Dame London: University of Notre Dame Press.

- McGoldrick, Dominic / Rowe, Peter / Donnelly, Eric (2004). The Permanent International Criminal Court. Legal and policy issues. Oxford and Portland (Oregon), Hart Publishing.

- Meyer, Lukas H. (editor) (2009). Legitimacy, justice and public international law. Cambridge: Cambridge University Press.

- Murphy, Colleen (2010). "Political reconciliation and international criminal trials". En May, Larry / Hoskins, Zachary: International criminal law and philosophy. Cambridge: Cambridge University Press.

- Olásolo, Héctor (2003). Corte Penal Internacional ¿Dónde investigar? Valencia: Tirant lo Blanch.

- Razesberger, Florian (2006). The International Criminal Court. Fráncfort del Meno: Peter Lang.

- Robinson, Darryl (2006). "Serving the interests of justice: Amnesties, truth commissions and the International Criminal Court". En Harrington, Joanna et al.: Bringing power to justice. Québec, McGill-Queen's University Press.

- Roht-Arriaza, Naomi (1995). "Punishment, redress, and pardon: theoretical and psychological approaches”. En Roht-Arriaza (editor): 
Impunity and Human Rights in International Law and Practice. New York oxford: Oxford University Press.

- Rotberg, Robert / Thomson, Dennis (editores) (2000). Truth versus justice. Princeton: Princeton University Press.

- Roxin, Claus (1998). Strafverfahrensrecht. Munich: Beck.

- Schabas; William (2010). The International Criminal Court: a commentary on the Rome Statute. Oxford: Oxford University Press.

- Scharf, Michael (1999): "The amnesty exception on the he jurisdiction of the International Criminal Court". Cornell International Law Journal (32).

- Stahn, Carsten (2005). "Complementarity, Amnesties and alternative forms of justice: some interpretative guidelines for the International Criminal Court". Journal of International Criminal Justice (3).

- Stigen, Jo (2008). The relationship between the International Criminal Court and National Jurisdictions: the principle of complementarity. Leiden Boston: Martinus Nijhoff Publishers.

- Struett, Michael J. (2009). "The politics of discursive legitimacy: understanding the dynamics and implications of prosecutorial discretion at the International Criminal Court". En Roach, Steven C.: Governance, order and the International Criminal Court. Oxford: Oxford University Press), pp. 107 y ss.

- Struett, Michael J. (2008). The Politics of constructing the International Criminal Court. New York: Palgrave Macmillan.

- Sunga, Lyal S. (2009). "Ten principles for reconciling truth commissions and criminal prosecutios". En Doria, José / Gasser, Hans-Peter / Bassiouni, M. Cherif: The legal regime of the International Criminal Court. Leiden. Boston: Martinus Nijhoff Publishers.

- Teitel, Ruti G. (2000). Transitional Justice. Oxford: Oxford University Press.

- Tomuschat, Christian (1998). "Das Statut von Rom für den Internationalen Strafgerichtshof". Die Friedenswarte.

- Valiña, Marta (2010). "Interpreting complementarity and Interests of justice in the presence of restorative-based alternative forms of justice". En Stahn, Carsten/Van den Herik, Larissa: Future perspectives of international criminal justice. La Haya: T.M.C. Asser Press.

- Villa-Vicencio, Charles (2000). "Restorative justice: dealing with the past differently". En Villa-Vicencio, Charles/Verwoerd, Wilhelm (editores): Looking back reaching forward. Reflections on the Truth and Reconciliation Commission of South Africa. Cape Town, London: University of Cape Town Press Zed. 
- Weвb, Philippa (2005). “The ICC Prosecutor's Discretion not to prosecute in the "interests of justice". Criminal Law Quarterly (50).

- WeI, Wu (2005). Die Rolle des Anklägers eines Internationales Strafgerichtshofs. Fráncfort del Meno: Peter Lang.

- Werle, Gerhard (2005). Tratado de derecho penal internacional. Valencia: Tirant lo Blanch.

- Wolfrum, Rüdeger / Röben, Volker (editores) (2008): Legitimacy in international law. Berlín, Heidelberg, Nueva York: Springer.

- Wouters, Jan / Verhoeven, Sten / Demeyer, Bruno (2009). “The International Criminal Court's Office of the Prosecutor". En Doria, José et al. The legal regime of the International Criminal Law. Leiden, Boston: Martinus Nijhoff Publishers.

- Yav Katshung, Joseph (2010): The International Criminal Courts and Truth Commissions: two sides of the same coin? Lexington: autoedición, pp. 38 y ss.

- Zahar, Alexander / Sluiter, Göran (2008). International criminal law. Oxford: Oxford University Press.

\section{NORMAS JURÍDICAS CITADAS}

- Pacto Internacional de Derechos Civiles y Políticos, adoptado y abierto a la firma, ratificación y adhesión por la Asamblea General en su resolución 2200 A (XXI), de 16 de diciembre de 1966 [fecha de consulta: 9 de enero de 2011]. Disponible en http://www2.ohchr. org/spanish/law/ccpr.htm

- Convención Interamericana sobre Derechos Humanos (Pacto de San José de Costa Rica) suscrita en la Conferencia especializada interamericana sobre derechos humanos, San José, Costa Rica 7 al 22 de noviembre de 1969 [fecha de consulta: 9 de enero de 2011]. Disponible en http://www.oas.org/juridico/spanish/tratados/b-32. html

- Convenio Europeo de los Derechos Humanos, suscrito en Roma el 4 de noviembre de 1950. Convenio para la Protección de los Derechos Humanos y de las Libertades Fundamentales revisado de conformidad con los Protocolos no 11 y 14 completado por el Protocolo adicional y los Protocolos no 4, 6, 7, 12 y 13 [fecha de consulta: 9 de enero de 2011]. Disponible en http:// www.echr.coe.int/NR/rdonlyres/1101E77A-C8E1-493F-809D800CBD20E595/0/ESP_CONV.pdf

- Convención de Viena sobre el Derecho de los Tratados Convención de Viena sobre el derecho de los tratados. Viena, 23 de mayo de 1969 [fecha de consulta: 9 de enero de 2011]. Disponible 
en http://untreaty.un.org/ilc/texts/instruments/english/ conventions/1_1_1969.pdf

- Estatuto de Roma de la Corte Penal Internacional [fecha de consulta: 9 de enero de 2011]. Disponible en http://www.icc-cpi.int/Menus/ ICC/Legal+Texts+and+Tools/

- Reglamento de la Corte Penal Internacional [fecha de consulta: 9 de enero de 2011]. Disponible en http://www.icc-cpi.int/Menus/ICC/ Legal+Texts+and+Tools/

- Regulations of the Office of the Prosecutor, Corte Penal Internacional [fecha de consulta: 9 de enero de 2010]. Disponible en http://www.icc-cpi.int/Menus/ICC/Legal+Texts+and+Tools/

- Reglas de Procedimiento y Prueba de la Corte Penal Internacional [Fecha de consulta: 9 de enero de 2010]. Disponible en http://www. icc-cpi.int/Menus/ICC/Legal+Texts+and+Tools/

- Código de conducta profesional de los abogados en la Corte Penal Internacional [fecha de consulta: 9 de enero de 2010]. Disponible en http://www.icc-cpi.int/Menus/ICC/Legal+Texts+and+Tools/

- Estatuto del Tribunal Penal Internacional para la ex Yugoslavia [fecha de consulta: 9 de enero de 2011]. Disponible en http://www.icty. org/sections/LegalLibrary

- Reglas de Procedimiento y Prueba del Tribunal Penal Internacional para la ex Yugoslavia [fecha de consulta: 9 de enero de 2011]. Disponible en http://www.icty.org/sections/LegalLibrary

- Estatuto del Tribunal Penal Internacional para Ruanda [fecha de consulta: 9 de enero de 2011]. Disponible en http://www.unictr.org/ tabid/94/default.aspx

- Estatuto de la Corte Especial para Sierra Leona [fecha de consulta: 9 de enero de 2011]. Disponible en http://www.sc-sl.org/LinkClick.as px?fileticket $=u$ Clnd $1 \mathrm{MJeEw} \% 3 \mathrm{~d} \&$ tabid $=176$

\section{JURISPRUDENCIA CITADA}

\section{Corte Penal Internacional}

- Situation in Darfur, Sudan (2009): Corte Penal Internacional 4 de febrero de 2009 (Decision on the application for leave to appeal the decision on application under rule 103) párrafo 29.

- Prosecutor v. Omar Hassan Ahmad Al Bashir (2009): Corte Penal Internacional 1 de septiembre de 2009 (Decision ordering the parties to submit their observations on the applications for Victims' participation in the proceedings) 
- Prosecutor $v$. Kony et al. (2008): Corte Penal Internacional 17 de septiembre de 2008 (Decision on legal representation, appointment of counsel of defence [...]).

- Prosecutor $v$. Germain Katanga (2008): Corte Penal Internacional, 10 de marzo de 2008 (Decision on the joinder of the Cases against Germain Katanga and Mathieu Ngudjolo Chui);

- Situation in the Democratic Republic of Congo (2006): Corte Penal Internacional 10 de febrero de 2006 (decision on the Prosecutor's application for warrants of arrest, article 58).

- Situation in the Central African Republic (2006): Corte Penal Internacional 30 de noviembre de 2006 (decision requesting information on the status of the preliminary examination of the situation in the Central African Republic).

\section{Tribunal Penal Internacional para la ex Yugoslavia}

- Prosecutor $v$. Prlic et al. (2004): Tribunal Penal Internacional para la ex Yugoslavia, 24 de noviembre de 2004 (decision on appeal by Bruno Stojic against Trial Chamber's decision on request for appointment of counsel);

- Prosecutor v. Milosevic (2004): Tribunal Penal Internacional para la ex Yugoslavia, 7 de diciembre de 2004 (decision on assigned counsel's motion for withdrawal);

- Prosecutor v. Hadzihasanovic et al. (2004): Tribunal Penal Internacional para la ex Yugoslavia, 11 de marzo de 2004 (decision on the prosecution motion for receiving testimony by videoconference link);

- Prosecutor $v$. Seselj (2003): Tribunal Penal Internacional para la ex Yugoslavia, 9 de mayo de 2003 (decision on presecution's motion for order appointing counsel to assist Vojislav Seselj with his defence);

- Prosecutor v. Musliu (2003): Tribunal Penal Internacional para la ex Yugoslavia, 21 de octubre de 2003 (decision on assignment of defence counsel);

- Prosecutor $v$. Jelisic (2000): Tribunal Penal Internacional para la ex Yugoslavia, 15 de noviembre de 2000 (decision on request to admit additional evidence);

- Prosecutor $v$. Sljivancanin (2003): Tribunal Penal Internacional para la ex Yugoslavia, 20 de agosto de 2001 (decision on assignment of defence counsel);

- Prosecutor $v$. Kupreskic et al. (2001): Tribunal Penal Internacional para la ex Yugoslavia, 11 de abril de 2001 (decision on the admission of additional evidence following hearing of 30 march 2001); 
- Prosecutor $v$. Blaojevic et al. (2001): Tribunal Penal Internacional para la ex Yugoslavia, 3 de julio de 2003 (decision on independent counsel for Vidoje Blagojevic's motion to instruct the registrar to appoint new lead and co-counsel);

- Prosecutor $v$. Simic et al. (2001): Tribunal Penal Internacional para la ex Yugoslavia, 24 de enero de 2001 (order separating proceedings and scheduling order).

- Prosecutor $v$. Tadic (1998): Tribunal Penal Internacional para la ex Yugoslavia, 15 de octubre de 1998 (decision on appellants motion for the extension of the time-limit and admission of additional evidence). 